\title{
ARTICLE
}

\section{Tailoring pH-Responsive Acrylic Acid Microgels with Hydrophobic Crosslinks for Drug Release}

\author{
B. Lu, ${ }^{a}$ M. D. Tarn, ${ }^{a}$ N. Pamme ${ }^{a, *}$ and T. K. Georgiou ${ }^{b, *}$,
}

Received ooth January 2012, Accepted ooth January 2012

DOI: 10.1039/xoxxooooox

www.rsc.org/
Amphiphilic microgels based on the hydrophilic acrylic acid (AA) and hydrophobic crosslinks of different compositions were synthesised using a lab-on-a-chip device. The microgels were formed by polymerising hydrophobic droplets. The droplets were generated via a microfluidic platform and contained a protected form of AA, a hydrophobic crosslinker (ethylene glycol dimethacrylate, EGDMA) and a free radical initiator in an organic solvent. Following photopolymerisation and subsequent hydrolysis, AA based microgels of amphiphilic nature were produced and it was demonstrated that they can successfully deliver both hydrophilic as well as hydrophobic moieties. The model drug delivery and the swelling ability of the microgels were influenced by the $\mathrm{pH}$ of the aqueous solution as well as the crosslinking density and hydrophobic content of the microgels.

\section{Introduction}

Microgels are gel particles with sizing ranging from 0.1 to $100 \mu \mathrm{m}$, and consisting of three-dimensionally crosslinked polymer networks. Such microgels have many exciting applications, including bioapplications in drug delivery, ${ }^{1-10}$ tissue engineering, ${ }^{2}$ and biosensing, ${ }^{11}$ as well as in applications and areas in the oil industry, ${ }^{12}$ organic dye removal, ${ }^{13}, 14$ coatings $^{15}$ and food industry ${ }^{16}$. Depending on the chemistry of the polymer chains, microgels can be dispersed in organic and/or aqueous solvents. For bio-applications like drug delivery it is important that the microgels are dispersed in water, i.e. they should contain hydrophilic components that will stabilise and disperse the microgels in water. However, it would be more advantageous if the microgels were amphiphilic, i.e. if they contained both hydrophobic and hydrophilic components, as they would be able to encapsulate and release both hydrophilic and hydrophobic drugs.

Amphiphilic microgels, however, are challenging to fabricate because most of the production methods, such as conventional emulsion templating, involve the formation of droplets in an immiscible continuous phase. The droplets contain pre-gels or polymerisable reagents that are later reacted to form the microgel. Thus, when fabricating hydrophilic microgels, the hydrophilic reagents are dispersed in the droplets while the continuous phase (CP) is an organic solvent in which the reagents have reduced or no solubility. The opposite applies when forming hydrophobic microgels; hydrophobic reagents are dispersed in the droplets while the $\mathrm{CP}$ is usually an immiscible aqueous solution. In order to fabricate amphiphilic microgels, one hydrophilic reagent and one hydrophobic would have to be stabilised and mixed together in a droplet without diffusing in the CP. However, if the CP is aqueous then the hydrophilic reagent will diffuse from the droplets to the $\mathrm{CP}$ and vice versa. If the $\mathrm{CP}$ is based on oil or an organic solvent, the hydrophobic reagents will diffuse from the droplets to the $\mathrm{CP}$. Thus, amphiphilic microgels are usually prepared using post- modifications ${ }^{1,4,7,17-21}$ and/or multi-step procedures. ${ }^{6,22}$ Commonly, hydrophilic microgels are fabricated and then modified to produce amphiphilic microgels by covalently or electrostatically binding amphiphilic or hydrophobic moieties. ${ }^{1,4,7,18-21}$

In this study we have used a microfluidic device to form droplets that were subsequently polymerised and hydrolysed to fabricate amphiphilic microgels that were based on acrylic acid (AA, ionisable and hydrophilic) and hydrophobic crosslinks based on the hydrophobic crosslinker ethylene glycol dimethacrylate (EGDMA). The application of a microfluidic platform offers the advantages of generating droplets, and consequently microgels, of narrower size distribution, while the size of the droplets can be tailored by varying the flow rates. ${ }^{16,23-26}$ Thus, the microgels are tailorable in terms of their size but also in terms of their composition since the crosslinking density, and so amphiphilicity, can also be varied by adjusting the molar ratio of the components. Finally, when using a microfluidic device immediate reactions following droplet generation can be performed, thereby enabling the polymerisation of some relatively unstable and easily hydrolysed monomers. For example, (meth)acrylic monomers when in contact with water tend to hydrolyze to (meth)acrylic acid, ${ }^{27}$ but (meth)acrylic acid will not be miscible in an organic solvent that contains another hydrophobic (macro)monomer or (macro)crosslinker in order to produce amphiphilic microgels.

Therefore, in order to produce amphiphilic microgels, a hydrophobic monomer that is a protected form of AA, specifically tetrahydropyranyl acrylate (THPA), was synthesised in-house and then copolymerised with the hydrophobic crosslinker, EGDMA, in chloroform droplets that were formed using microfluidic devices. The protective group of tetrahydropyranyl was chosen because is a good leaving group and it can be easily be hydrolysed even in macrogels. ${ }^{28-31}$ The hydrolysis of the THPA groups produced anionic gels and we have chosen anionic gels because they have shown promised in drug delivery ${ }^{32}$ and protein delivery ${ }^{33}$. Specifically in 
this study microgels of differing crosslinking ratios were produced, collected and polymerised off the chip and then hydrolysed to produce AA based microgels. The $\mathrm{pH}$ responsiveness of the AA based microgels was evaluated by studying their swelling in different $\mathrm{pH}$ environments. Finally, to prove that the amphiphilic nature of the microgels assists in the delivery of drugs of different hydrophilicities, the encapsulation and delivery of both hydrophobic and hydrophilic moieties was investigated. To the best of our knowledge, this is the first report of covalently linked microgels of amphiphilic nature that have been prepared using a microfluidic platform.

\section{Experimental}

\section{Materials and Methods}

Acrylic acid (AA, 99 \%), 2,3-dihydro-2H-pyran (DHP, $99 \%$ ), ethylene glycol dimethacrylate (EGDMA), sodium dodecyl sulfate

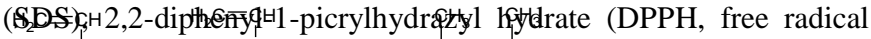

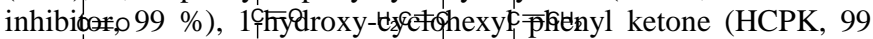
$\%$, fre radical initiator) as the free-radfeal initiator, sudan I, trypan

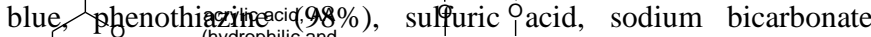
$\mathrm{NaHCO} 3$, sodiuph-reqdphanate $\mathrm{Na}_{2} \mathrm{SG}_{4} \mathrm{H}_{2}$ hy 9 lrochloric acid and sodium hydroxide and basicmartumina wertiverpurfebased from Sigma-Aldrich

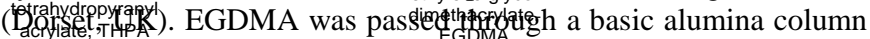
to (reckppeperic the free radical initiatrofropuriber use. The organic solvent us monomer, and was purchased from Fisher Scientific (Loughborough, UK). The chemical structure of the main reagents used (AA and EGDMA) and the in-house synthesised monomer (synthesis described below) are shown in Fig. 1.<smiles>C=CC(=O)OC1CCCCO1</smiles>

tetrahydropyranyl acrylate, THPA (hydrophobic monomer, protected AA)<smiles>C=CC(=O)O</smiles>

acrylic acid, AA (hydrophilic and $\mathrm{pH}$-responsive monomer)<smiles>C=C(C)C(=O)OCCOC(=O)C(=C)C</smiles>

ethylene glycol dimethacrylate, EGDMA (hydrophobic crosslinker)
Figure 1. Chemical structures of the monomers and crosslinker.

Synthesis of Protected Acrylic Acid Monomer, Tetrahydropyranyl Acrylate (THPA)

The synthesis of THPA was similar to the previously described synthesis of tetrahydropyranyl methacrylate. ${ }^{28-30,34,35} \mathrm{In}$ a $500 \mathrm{~mL}$ three neck round bottom flask that contained $1 \mathrm{~g}$ of phenothiazine (free radical inhibitor) and $125 \mathrm{~mL}$ of DHP (116.25 g, $1.38 \mathrm{~mol}), 20$ drops of a $50 \% \mathrm{v} / \mathrm{v}$ sulfuric acid solution in water were added dropwise. The colour should change to brown. Following this, a mixture of $125 \mathrm{~mL}$ DHP (116.25 g, $1.38 \mathrm{~mol})$ and $94.6 \mathrm{~mL} \mathrm{AA} \mathrm{(99.4} \mathrm{g,} 1.38$ $\mathrm{mol}$ ) and $1 \mathrm{~g}$ phenothiazine (using a dropping funnel) was added and the round bottom flask was placed in an oil bath. The mixture was then left to react overnight at $50{ }^{\circ} \mathrm{C}$.

The next day, once the mixture had cooled down, $10 \mathrm{~g}$ of $\mathrm{NaHCO}_{3}$ and $40 \mathrm{~g}$ of $\mathrm{Na}_{2} \mathrm{SO}_{4}$ were added and allowed to stir for 2-3 h. The solids were then filtered out and the monomer mixture was passed through basic alumina column twice (to remove the un-reacted AA).
The removal of AA was confirmed by ${ }^{1} \mathrm{H}$ NMR. A free radical inhibitor, DPPH, was added into the THPA monomer and the monomer it was stored in the fridge. It was distil under vacuum prior to use.

\section{Fabrication of Microgels}

Microfluidic Chip Fabrication and Setup

THPA-EGDMA containing droplets were generated in a flow focusing chip geometry with a CP inlet, a dispersed phase (DP) inlet, and a single straight outlet channel (Fig. 2a). The channel structure was etched in glass to a depth of $50 \mu \mathrm{m}$ via photolithography and wet etching techniques, and the channels featured a width of $150 \mu \mathrm{m}$ following the etching process ${ }^{36}$ Fused silica capillaries $(150 \mu \mathrm{m}$ i.d., $363 \mu \mathrm{m}$ o.d., CM Scientific, UK) were glued into the inlet and outlet holes and connected to $500 \mu \mathrm{L}$ glass syringes (SGE, Sigma-Aldrich, UK) by syringe adaptors (IDEX Health \& Science, UK) (Fig. 2b). Two syringe pumps (PHD2000, Harvard Apparatus, UK) were used to control the flow rates of the $\mathrm{CP}$ and DP between $0.5 \mu \mathrm{L} \mathrm{min}^{-1}$ and $50 \mu \mathrm{L} \mathrm{min}{ }^{-1}$. A $5 \mathrm{~cm}$ long piece of Tygon tubing $(254 \mu \mathrm{m}$ i.d., 762 $\mu \mathrm{m}$ o.d., Cole-Parmer, UK) was attached to the outlet capillary at one end while the other end was placed onto a microscope slide or a Petri dish for droplet collection. Droplets were photographed with a colour CCD camera (MTV-63V1N, Mintron, Taiwan) attached to an inverted microscope (Eclipse Ti, Nikon, UK), with images captured using WinDVD Creator 2 (Corel Ltd., UK) software. ImageJ freeware was used for the analysis of droplet size and colour intensity. Photopolymerisation was achieved using a $15 \mathrm{~W}$ UV light source (XX-15S, Ultra-Violet Products Ltd., UK). The entire setup was covered with a thick black cloth to in order to protect users from UV irradiation. Chips and syringes were covered with aluminum foil to prevent the reagents from gelating prior to their collection as droplets.

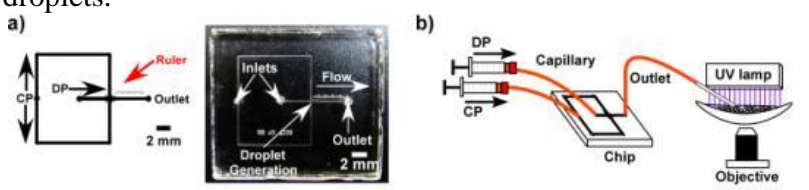

Figure 2: (a) Layout and photograph of the flow focusing chip employed for microgel precursor droplet formation. The design featured a central channel for the oil based DP, containing the reagents, and outer channels for the $\mathrm{CP}$, which merged into a $5 \mathrm{~mm}$ long outlet channel. (b) Experimental setup for on-chip droplet generation and collection of the droplets onto a slide or dish for UV initiated photopolymerisation.

\section{Formation of Droplets and Polymerisation of Microgels}

The DP was based on chloroform containing THPA and EGDMA with varying molar ratios (though the total concentration of the two reagents was always $30 \mathrm{wt} \%$ ), and $4 \mathrm{wt} \%$ of HCPK (free radical photo-initiator). The $\mathrm{CP}$ was an aqueous solution of $0.1 \mathrm{wt} \%$ SDS. The concentration of SDS was below the critical micelle concentration. $^{37}$ The flow rates of the $\mathrm{CP}$ and DP were $5 \mu \mathrm{L} \mathrm{min}{ }^{-1}$ and $0.5 \mu \mathrm{L} \mathrm{min}{ }^{-1}$, respectively, and were chosen following preliminary results which showed that stable droplets were formed at these flow rates. After droplet formation in the microfluidic device, the droplets were collected on a microscope slide or Petri dish and polymerised by UV irradiation for $30 \mathrm{~min}$. Three THPA-EGDMA microgels of differing compositions were formed, with molar ratios of 30:4, 50:4, and 70:4 THPA:EGDMA. The polymerised microgels were then hydrolysed in $1 \mathrm{M} \mathrm{HCl}$ overnight to produce $\mathrm{pH}$ responsive AA-EGDMA microgels. The hydrolysis was confirmed with FTIR.

\section{Swelling Studies in Different $\mathrm{pH}$ environments}

Aqueous solutions of $\mathrm{NaOH}(1 \mathrm{M})$ and $\mathrm{HCl}(1 \mathrm{M})$ were used to vary the $\mathrm{pH}$ of the solution that the AA-EGDMA microgels were 
suspended in, from $\mathrm{pH} 1$ to $\mathrm{pH} 14$, in order to study the responsiveness of the microgel particles. The microgels were photographed with the colour CCD camera and inverted microscope setup described previously, with ImageJ used for image analysis. The extent of swelling and shrinking (relative size) was calculated by dividing the microgel size at a given $\mathrm{pH}$ by their original size measured at $\mathrm{pH} 7$.

\section{pK $K_{a}$ Determination}

The $p \mathrm{~K}_{\mathrm{a}}$ of the three synthesised microgels was determined by potentiometric titration. Specifically $0.02 \mathrm{~g}$ of each microgel was dispersed in water, $1 \mathrm{M}$ of $\mathrm{NaOH}$ was added to increase the $\mathrm{pH}$ above $11 \mathrm{M}$ and then the titration was performed by adding $0.05 \mathrm{~mL}$ of $0.1 \mathrm{M} \mathrm{HCl}$ and measuring the $\mathrm{pH}$ after each addition.

\section{Dye Encapsulation and Release Studies}

Two types of dye were encapsulated in the microgels via two different methodologies, in order to determine the capability of the microgels to encapsulate and release both hydrophilic and hydrophobic moieties. $0.5 \mathrm{wt} \%$ hydrophobic Sudan I was mixed into the DP when the droplets were being prepared in the microfluidic channel, hence the dye was already present inside the microgel following the polymerisation step. $0.5 \mathrm{wt} \%$ hydrophilic Trypan Blue was loaded into already polymerised AA-EGDMA microgels in a $\mathrm{pH} 14$ solution by diffusion with sonication. The chemical structures of both dyes are shown in Fig. 3. The model drug release was monitored using the colour camera of the microscope, in particular how the colour intensity of the microgels changed during release. Images were taken at regular time intervals (every $1 \mathrm{~min}$ or $5 \mathrm{~min}$ depending how fast the release was). ImageJ freeware was used for the analysis of the colour intensity.

(b)

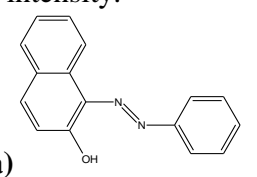

(a)

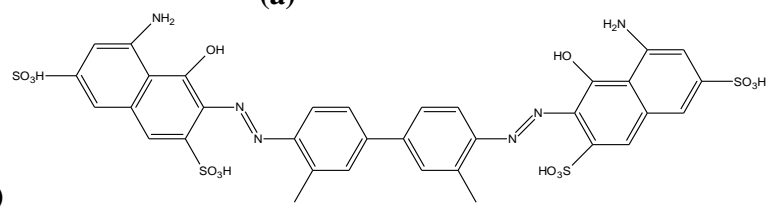

Figure 3. Chemical structures of (a) hydrophobic Sudan I, and (b) hydrophilic Trypan Blue.

\section{Results and discussion}

\section{Microgel Fabrication and pH Responsiveness Studies}

Three types of amphiphilic AA-EDGMA microgels of different crosslinking densities and hydrophobic contents were successfully fabricated via a microfluidics approach. Specifically, droplets of THPA:EGDMA in chloroform were formed in an aqueous CP and collected in on a slide or dish, then polymerised via UV-initiated free radical polymerisation. Following polymerisation, these THPAEGDMA microgels were hydrolysed by suspending them in a $\mathrm{pH} 1$ solution to produce AA-EGDMA microgels. The hydrolysis step caused the microgels to shrink due to protonation of the ionisable AA units. For example, when the 50:4 THPA-EGDMA microgel (Fig. 4a) was hydrolysed to AA-EGDMA (Fig. 4b) the microgel shrank from $131 \mu \mathrm{m}$ to $98 \mu \mathrm{m}$. However, when the AA-EGDMA microgel was then suspended in a pH 14 solution it swelled to 203 $\mu \mathrm{m}$.

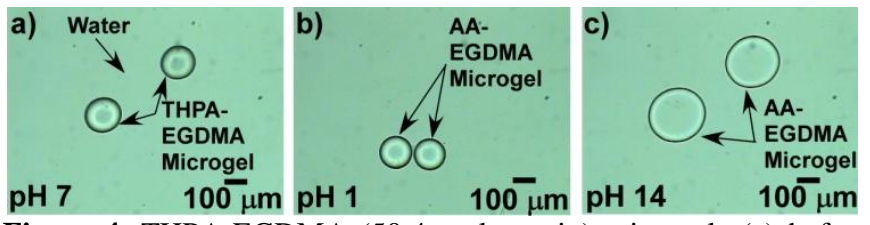

Figure 4. THPA-EGDMA (50:4 molar ratio) microgels (a) before hydrolysis at $\mathrm{pH} \mathrm{7,} \mathrm{(b)} \mathrm{after} \mathrm{hydrolysis} \mathrm{(forming} \mathrm{AA-EGDMA}$ microgels) at $\mathrm{pH}$, and (c) at $\mathrm{pH} 14$.

The $\mathrm{pH}$ responsiveness of each type of AA-EGDMA microgels formed with each molar ratio of THPA:EGDMA (30:4, 50:4, and $70: 4)$ was studied by measuring the size of the microgels at different $\mathrm{pH}$ values. As can be observed in Fig. 5, the size of the microgels increased with the increase in the $\mathrm{pH}$ of the solution. This was expected and it was due to the ionisable and hydrophilic AA units of the microgels as it was confirmed by the microgels $\mathrm{p} K_{\mathrm{a}} \mathrm{s}$ that were determined with potentiomentic titration. Specifically the $\mathrm{p} K_{\mathrm{a}} \mathrm{s}$ were found to be $6.1,6.2$ and 6.2 for the three microgels with different AA:EGDMA ratios, 30:4, 50:4, and 70:4, respectively, similar to that of pAA based stars. ${ }^{38}$ Hence the AA units are negatively charged at high $\mathrm{pH}$ due to deprotonation, while they are not ionised at low $\mathrm{pH}$ because they are protonated. Thus, at high $\mathrm{pH}$ values the polymer chains expand and extend due to the repulsive forces caused by the negatively charged AA units. When the polymer chains expand the entire microgel structure expands/swells. Returning the microgels to a lower $\mathrm{pH}$ environment causes them to shrink again as a result of deprotonation. This reversible $\mathrm{pH}$ responsive behaviour was expected, and has been observed previously for (meth)acrylic acid containing microgels $\mathrm{s}^{4,5,39}$ and macrogels, ${ }^{29,} 30,34$ and this property makes the AA-EGDMA microgels promising for encapsulation and release of various moieties. Note that the size of the microgels at very high $\mathrm{pH}$ reduces because of increased ionic strength due to the increase $\mathrm{NaOH}$ concentration as it has been observed in macrogels. ${ }^{29,30}$

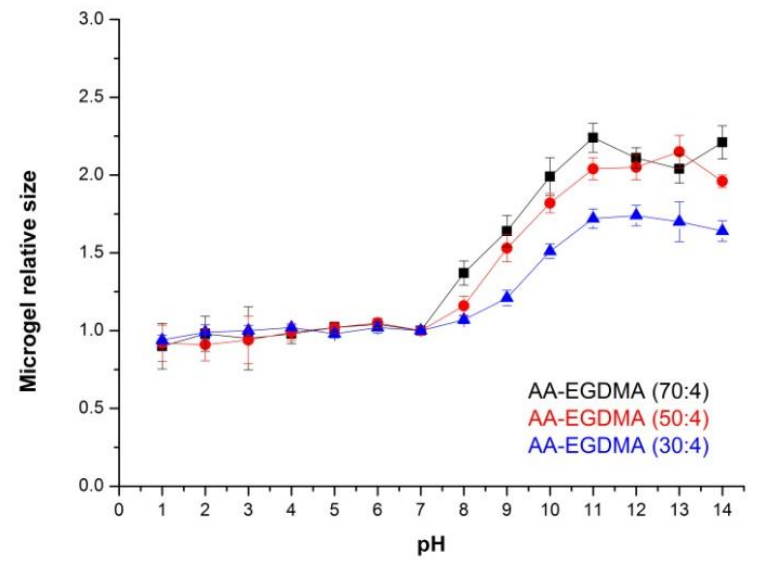

Figure 5. Swelling ratios of the AA-EGDMA microgels in different $\mathrm{pH}$ environments. Microgels formed from THPA:EGDMA molar ratiosof 70:4, 50:4 and 30:4 are represented as squares, circles and triangles, respectively.

When comparing the three types of microgels in Fig. 5, it was apparent that by increasing the crosslinking density and the hydrophobic content, the size of the microgels decreased, as anticipated since the crosslinks avert the microgel from expanding. This expected trend, that has been observed before in microgels ${ }^{4,39}$ as well as macrogels, ${ }^{40}$ was only apparent in high $\mathrm{pH}$ solutions in which the AA units were ionised. At lower $\mathrm{pH}$ values, the polymer chains of the microgels were not extended, i.e. the microgels were in 
a collapsed (shrunken) state, so the crosslinks no longer interfered with the polymer chain movement and expansion.

\section{Dye Encapsulation and Release Studies}

A hydrophobic and a hydrophilic dye were encapsulated and released to order to prove that the amphiphilic nature of the AAEGDMA microgels could be utilised for the capture and delivery of moieties of varying hydrophobicity/hydrophilicity, similarly to amphiphilic macrogels ${ }^{41,42}$. The release of the model drugs were trigged by making the $\mathrm{pH}$ of the environment basic such that the AA units became ionised and the microgels swelled, allowing the dyes to diffuse out.

Hydrophobic Dye - Sudan I. Sudan I (see the chemical structure in Fig. 3a) was encapsulated into the microgel by adding it to the oil based DP for droplet formation, meaning that the dye was already present in the microgels when they were then polymerised. THPAEGDMA (50:4 molar ratio) droplets containing the Sudan I are shown in Fig. 6a, while Fig. 6b shows the microgels following polymerisation. The THPA-EGDMA microgels were hydrolysed in $\mathrm{pH} 1$ solution to form AA-EGDMA microgels. When the 50:4 AAEGDMA microgels were dispersed in $\mathrm{pH} 14$ solution, as shown in Fig. $6 c$, the dye, which appears orange in the pictures although the colour is actually red, can clearly be seen diffusing out of the microgel. As described in the previous section, when suspended in basic solution the microgel swells due to extension of the polymer chains via repulsive ionic interactions, thereby enlarging the pores in the microgels and allowing the dye to be released. Furthermore, the AA units become more hydrophilic when they are transferred from acidic to basic $\mathrm{pH}$, and so they are no longer able to retain the hydrophobic dye.
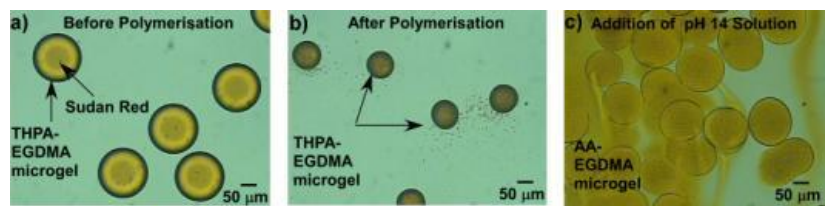

Figure 6. (a) 50:4 THPA-EGDMA microgels with hydrophobic Sudan I dye encapsulated during droplet generation. (b) 50:4 THPAEGDMA microgels after polymerisation $(\mathrm{pH}=7)$. (c) Sudan I dye release from 50:4 AA-EGDMA microgels in $\mathrm{pH} 14$ solution.

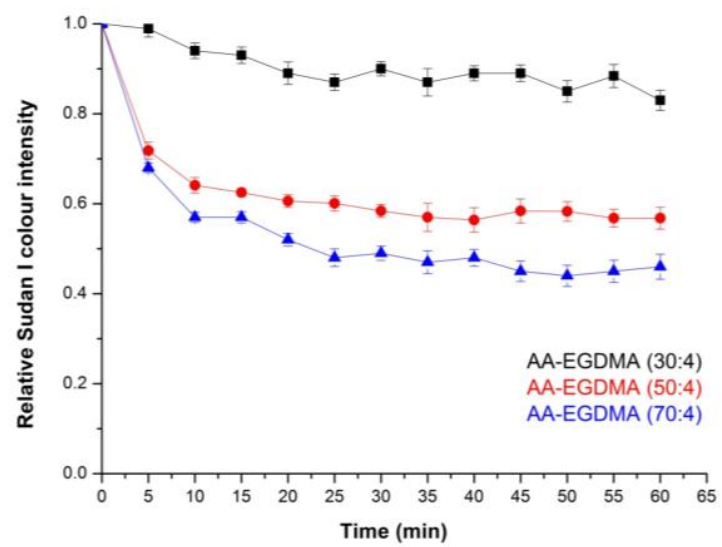

Figure 7. Hydrophobic drug release at basic $\mathrm{pH}(\mathrm{pH}>11)$. The plots show the relative Sudan I colour intensity versus time for all three types of AA-EGDMA microgels with different crosslinking densities. Squares, circles and triangles represent the 70:4, 50:4 and 30:4 AA:EGDMA molar ratios, respectively.

The release of the hydrophobic model drug was further evaluated by studying the release of Sudan I over time for all three types of microgels. The results are shown in Fig. 7. As can be clearly observed, the crosslinking density and hydrophobic content of the microgel strongly affects the release of Sudan I. Specifically, by increasing the crosslinking density (EGDMA content) the rate, as well as the amount, of hydrophobic dye released is reduced. This was expected, since by increasing the crosslinking density the mesh/pore size of the microgels decreases ${ }^{43,44}$ so it is more difficult for the dye to diffuse out. In addition, by increasing the EGDMA (crosslinker) content, which is itself hydrophobic, the hydrophobic dye will be retained in the microgel structure to a great extent due to van der Waal's interactions.

Hydrophilic Dye - Trypan Blue. A hydrophilic dye, Trypan Blue (see the chemical structure in Fig. 3b), was also investigated. In this case, $0.5 \mathrm{wt} \%$ Trypan Blue was loaded into the AA-EGDMA microgels after they had already been polymerised and hydrolysed. Thus was achieved by suspending the AA-EGDMA microgels into $\mathrm{pH} 14$ solution in order to make them swell, allowing gradual diffusion of the Trypan Blue into the microgels which was assisted by sonication. Figure $8 \mathrm{a}$ shows this process for 50:4 molar ratio AA-EGDMA microgels. As the $\mathrm{pH}$ was then reduced to $\mathrm{pH} 1$, the microgel shrank and the Trypan Blue was encapsulated and concentrated within the gel particle. After purification, the $\mathrm{pH}$ was increased again to $\mathrm{pH} 7$ (Fig. 8b), where it was found that the hydrophilic Trypan Blue was gradually released into the surrounding water. It can be seen that the blue colour intensity inside the microgel faded within an hour (Fig. $8 \mathrm{c}$ ), indicating release of the dye from the microgel.
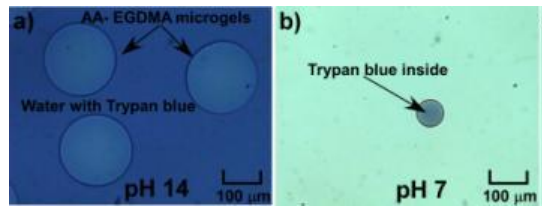

c) After an hour

Figure 8. Hydrophilic Trypan Blue encapsulation in, and release from, 50:4 AA-EGDMA microgels. (a) Encapsulation of the dye in $\mathrm{pH} 14$ solution via diffusion. (b) Microgels containing the Trypan Blue immediately after their introduction into a $\mathrm{pH} 7$ environment, and (c) after an hour of dye release at $\mathrm{pH} 7$.

The release of the hydrophilic dye was further investigated over time for all three types of AA-EGDMA microgels in a basic $\mathrm{pH}$ solution $(\sim \mathrm{pH} 8.5)$. The results are shown in Fig. 9, and it is apparent that the release of Trypan Blue was influenced by the crosslinking ratio in a similar manner to the release of the hydrophobic dye (Sudan I). In particular, by increasing the crosslinking density and thus reducing the mesh size, the release of the hydrophilic dye was slower. This was expected since the higher mesh/pore size allows the dye to diffuse more easily and faster through the microgel structure.

Compared to the hydrophobic Sudan I release, the release of Trypan Blue from the AA-EGDMA microgels was much faster for all three types of microgel. For example, it took more than $25 \mathrm{~min}$ for the release of the hydrophobic Sudan I from the 70:4 AA-EGDMA microgel at basic $\mathrm{pH}$ to reach a plateau, while the plateau was reached in only 5 minutes when the hydrophilic Trypan Blue dye was being released. This can be attributed to the difficulty of the hydrophobic dye to diffuse into an aqueous environment compared to the hydrophilic dye as well as the fact that the hydrophobic domains of the microgels retain for longer the hydrophobic dye through van der Waals interactions.

In summary, the AA-EGDMA microgels were able to encapsulate and deliver both hydrophobic and hydrophilic dyes, and it has been demonstrated that the rate of release can be varied by adjusting the 
crosslinking density and hydrophobic content of the microgel.

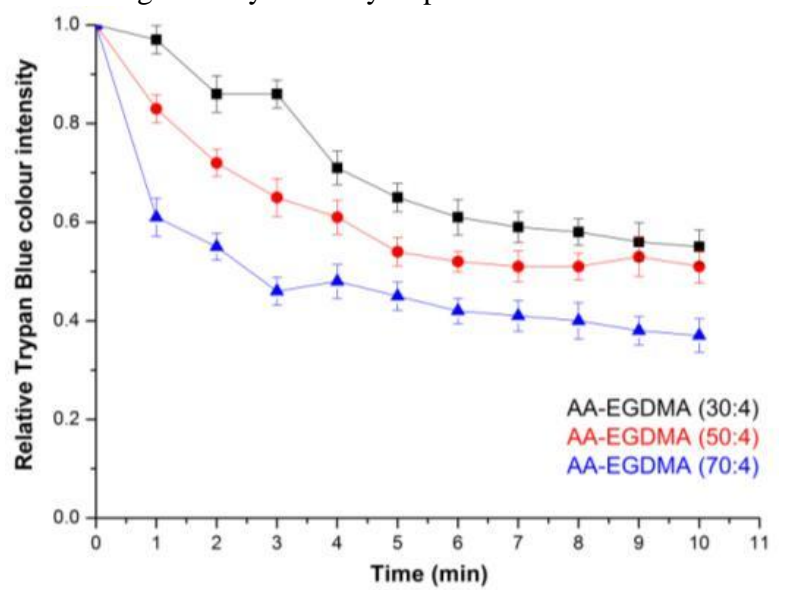

Figure 9. Hydrophilic dye release in basic $\mathrm{pH}$ solution $(\sim \mathrm{pH} 8.5)$. The plots show the relative Trypan Blue colour intensity versus time for all three types of AA-EGDMA microgels with different crosslinking densities. Squares, circles and triangles represent the 70:4, 50:4 and 30:4 AA:EGDMA molar ratios, respectively.

\section{Conclusions}

Amphiphilic microgels based on hydrophilic, ionisable acrylic acid (AA) and the hydrophobic crosslinker ethylene glycol dimethacrylate (EGDMA) were successfully synthesised from droplets formed on a microfluidic platform. The use of microfluidics enabled the systematic variation of the crosslinking density and the synthesis of microgels with narrower size distributions than those prepared by conventional methods. The microgels were $\mathrm{pH}$ responsive. By increasing the $\mathrm{pH}$ of the surrounding solution, the size of the microgels increased due to ionisation of the AA units and the resultant electrostatic repulsion between them inside the gel particles. The swelling of the microgels increased by decreasing the crosslinking density. Finally, the encapsulation and release of a hydrophobic and a hydrophilic dye, which were used as model drugs, was investigated. The hydrophobic dye was incorporated during the droplet generation stage, while the hydrophilic dye was absorbed by microgels that had already been polymerised. The subsequent delivery was investigated and it was proven that the $\mathrm{pH}$ can be used to trigger the release. Thus, the amphiphilic nature of the AA-EGDMA microgels allowed the encapsulation and delivery of both types of model drugs, and as expected the hydrophilic dye was released faster than the hydrophobic dye since it was able to diffuse faster through the microgel and into the aqueous surroundings.

\section{Acknowledgements}

University of Hull is thanked for funding $\mathrm{Mr} \mathrm{Lu}$ 's $\mathrm{PhD}$ studentship.

\section{Notes and references}

${ }^{a}$ Department of Chemistry, University of Hull, HU6 7RX, Hull, UK.

${ }^{b}$ Department of Materials, Imperial College London, SW7 2AZ, London, UK.

1. V. Alakhov, G. Pietrzynski, K. Patel, A. Kabanov, L. Bromberg and T. A. Hatton, J. Pharm. Pharmacol., 2004, 56, 1233-1241.
2. Y. Jiang, J. Chen, C. Deng, E. J. Suuronen and Z. Zhong, Biomaterials, 2014, 35, 4969-4985.

3. Y. Gao, A. Ahiabu and M. J. Serpe, ACS Appl. Mater. Interfaces, 2014, 6, 13749-13756.

4. L. Bromberg, M. Temchenko and T. A. Hatton, Langmuir, 2002, 18, 4944-4952.

5. J. P. K. Tan and K. C. Tam, J. Controlled Release, 2007, 118, 87-94.

6. W. C. Lee, Y. C. Li and I. M. Chu, Macromol. Biosci., 2006, 6, 846854.

7. L. Li, C. Cheng, M. P. Schürings, X. Zhu and A. Pich, Polymer, 2012, 53, 3117-3123.

8. M. Dadsetan, K. E. Taylor, C. Yong, Ž. Bajzer, L. Lu and M. J. Yaszemski, Acta Biomaterialia, 2013, 9, 5438-5446.

9. H. Bysell, R. Månsson, P. Hansson and M. Malmsten, Adv. Drug Delivery Rev., 2011, 63, 1172-1185.

10. T. Trongsatitkul and B. M. Budhlall, Polym. Chem., 2013, 4, 15021516.

11. M. R. Islam, A. Ahiabu, X. Li and M. J. Serpe, Sensors, 2014, 14, 8984-8995.

12. Y. Chen, Y. Bai, S. Chen, J. Jup, Y. Li, T. Wang and Q. Wang, ACS Appl. Mater. Interfaces, 2014, 6, 13334-13338.

13. D. Parasuraman and M. J. Serpe, ACS Appl. Mater. Interfaces, 2011, 3, 4714-4721.

14. D. Parasuraman and M. J. Serpe, ACS Appl. Mater. Interfaces, 2011, 3, 2732-2737.

15. C. D. Sorrell, M. C. D. Carter and M. J. Serpe, ACS Appl. Mater. Interfaces, 2011, 3, 1140-1147.

16. H. M. Shewan and J. R. Stokes, J. Food Eng., 2013, 119, 781-792.

17. Anon, Macromolecules, 1990, 23, 5158-5160.

18. L. Bromberg, M. Temchenko, G. D. Moeser and T. A. Hatton, Langmuir, 2004, 20, 5683-5692.

19. X. Wang, X. Hou, Y. Wu and S. You, J. Appl. Polym. Sci., 2009, 114, 4042-4050.

20. H. Li, X. Cui, S. Shen and D. Hu, J. Appl. Polym. Sci., 2011, 122, 509-516.

21. H. Li, P. Zhang, L. Zhang, T. Zhou and D. Hu, J. Mater. Chem., 2009, 19, 4575-4586.

22. T. Zhou, H. Li, G. Liu, L. Zhang, D. Yao and D. Hu, J. Appl. Polym. Sci., 2009, 114, 4000-4010.

23. E. Tumarkin and E. Kumacheva, Chem. Soc. Rev., 2009, 38, 21612168.

24. S. Seiffert and D. A. Weitz, Polymer, 2010, 51, 5883-5889.

25. W. J. Duncanson, T. Lin, A. R. Abate, S. Seiffert, R. K. Shah and D. A. Weitz, Lab on a Chip - Miniaturisation for Chemistry and Biology, 2012, 12, 2135-2145.

26. S. Seiffert, Angew. Chem. - Int. Ed., 2013, 52, 11462-11468.

27. P. Van De Watering, N. J. Zuidam, M. J. Van Steenbergen, O. A. G. J. Van Der Houwen, W. J. M. Underberg and W. E. Hennink, Macromolecules, 1998, 31, 8063-8068.

28. S. N. Georgiades, M. Vamvakaki and C. S. Patrickios, Macromolecules, 2002, 35, 4903-4911.

29. G. Kali, T. K. Georgiou, B. Iván, C. S. Patrickios, E. Loizou, Y. Thomann and J. C. Tiller, Langmuir, 2007, 23, 10746-10755.

30. G. Kali, T. K. Georgiou, B. Iván, C. S. Patrickios, E. Loizou, Y. Thomann and J. C. Tiller, Macromolecules, 2007, 40, 2192-2200. 
31. G. Kali, T. K. Georgiou, B. Iván and C. S. Patrickios, J. Polym. Sci., Part A: Polym. Chem., 2009, 47, 4289-4301.

32. X. Hu, W. Wei, X. Qi, H. Yu, L. Feng, J. Li, S. Wang, J. Zhang and W. Dong, J. Mater. Chem. B, 2015, 3, 2685-2697.

33. B. Kim, S. H. Lim and W. Ryoo, J. Biomater. Sci., Polym. Ed., 2009, 20, 427-436.

34. T. K. Georgiou, L. A. Phylactou and C. S. Patrickios, Biomacromolecules, 2006, 7, 3505-3512.

35. K. S. Pafiti, Z. Philippou, E. Loizou, L. Porcar and C. S. Patrickios, Macromolecules, 2011, 44, 5352-5362.

36. T. McCreedy, Analytica Chimica Acta, 2001, 427, 39-43.

37. A. Pal and S. Chaudhary, Fluid Phase Equilibria, 2014, 372, 100104.

38. F. A. Plamper, H. Becker, M. Lanzendörfer, M. Patel, A. Wittemann, M. Ballauff and A. H. E. Müller, Macromol. Chem. Phys., 2005, 206, 1813-1825.

39. G. M. Eichenbaum, P. F. Kiser, A. V. Dobrynin, S. A. Simon and D. Needham, Macromolecules, 1999, 32, 4867-4878.

40. M. Haraszti, E. Tóth and B. Iván, Chem. Mater., 2006, 18, 49524958.

41. P. Papaphilippou, M. Christodoulou, O. M. Marinica, A. Taculescu, L. Vekas, K. Chrissafis and T. Krasia-Christoforou, ACS Appl. Mater. Interfaces, 2012, 4, 2139-2147.

42. N. Ghasdian, E. Church, A. P. Cottam, K. Hornsby, M. Y. Leung and T. K. Georgiou, RSC Advances, 2013, 3, 19070-19080.

43. A. W. Chan and R. J. Neufeld, Biomaterials, 2009, 30, 6119-6129.

44. R. Suriano, G. Griffini, M. Chiari, M. Levi and S. Turri, J. Mech. Behav. Biomed. Mater., 2014, 30, 339-346. 